\title{
Superconductivity in the two-band Hubbard model
}

\author{
Akihisa Koga \\ Department of Physics, Tokyo Institute of Technology, Tokyo 152-8551, Japan \\ Philipp Werner \\ Department of Physics, University of Fribourg, 1700 Fribourg, Switzerland
}

(Received 4 December 2014; revised manuscript received 25 January 2015; published 12 February 2015)

\begin{abstract}
We study the two-band Hubbard model in infinite dimensions by solving the dynamical mean-field equations with a strong coupling continuous-time quantum Monte Carlo method and show that an $s$-wave superconducting state can be stabilized in the repulsively interacting case. We discuss how this superconducting state competes with the metallic and paired Mott states. The effects of the Hund coupling and crystalline electric field are also addressed.
\end{abstract}

DOI: 10.1103/PhysRevB.91.085108

PACS number(s): 71.10.Fd, 74.25.Dw, 71.30.+h

\section{INTRODUCTION}

Strongly correlated electron systems with orbital degrees of freedom are attracting much interest in condensed matter physics. A particularly active topic is the study of superconducting (SC) states in transition-metal oxides such as $\mathrm{Sr}_{2} \mathrm{RuO}_{4}$ [1], $\mathrm{La}\left(\mathrm{O}_{1-\mathrm{x}} \mathrm{F}_{\mathrm{x}}\right) \mathrm{FeAs}$ [2], $\mathrm{Na}_{\mathrm{x}} \mathrm{CoO}_{2}$ [3], and fullerene-based solids $A_{3} \mathrm{C}_{60}(A=$ alkali metal) [4-6]. These phenomena have been investigated theoretically by taking into account Coulomb interactions, Hund coupling, the multiband structure, and the lattice geometry. Electron correlations have been treated by means of various analytical and numerical methods such as the random phase approximation, fluctuationexchange approximation, and dynamical cluster approximation. While the relevant diagrams should be included in the method, it may be difficult to identify which ones are crucial for stabilizing the SC state. A simple question that naturally arises is whether or not a SC state can be realized in a model with repulsive interactions, without any particular properties of the band structure. Since the onsite Coulomb repulsion usually prevents the formation of tightly coupled singlet pairs, it is expected that an unconventional superconducting state with an anisotropic gap should be realized instead of the $s$-wave superconducting state. This simple argument should be valid in the single band case.

In multiorbital systems, on the other hand, the situation may be more complicated. Capone et al. have studied the two-band Hubbard model with intraorbital interaction $U$, interorbital interaction $U^{\prime}$ and Hund coupling $J[7,8]$. They showed that a strongly correlated SC ground state appears under certain conditions when $U=U^{\prime}$ and $J<0$. However, in this analysis, the role of the Hund coupling is not quite clear since it is naively expected that the interorbital exchange interaction suppresses intraorbital pairing correlations. Therefore, it is instructive to clarify which interactions stabilize the superconducting state in the two-orbital system. Furthermore, it is interesting to explore whether or not the SC state in the strongly correlated region is adiabatically connected to the trivial BCS-type SC state stabilized by weak attractive interactions. This may give us a simple explanation for the stability of the SC state in the repulsively interacting system.

To clarify these issues, we consider the two-orbital Hubbard model. By combined use of dynamical mean-field theory
(DMFT) [9-12] and the continuous-time quantum Monte Carlo (CTQMC) method [13,14], we show that if orbital and magnetic ordering is suppressed, an $s$-wave SC state is realized in the model with degenerate orbitals at half-filling, and that it is adiabatically connected to the simple BCS-type SC state. The effect of the crystalline electric field is also addressed.

The paper is organized as follows. In Sec. II, we introduce the two-band Hubbard model and briefly summarize our theoretical approach. In Sec. III, we demonstrate the existence of a SC state in the repulsively interacting region of parameter space. We study the stability of the $s$-wave SC state at low temperatures in Sec. IV. A brief summary is given in the last section.

\section{MODEL AND METHOD}

We consider a correlated electron system with two orbitals, which is described by the Hubbard Hamiltonian [15-23]

$$
\begin{aligned}
H= & \sum_{\substack{\langle i, j\rangle \\
\alpha, \sigma}} t_{i j} c_{i \alpha \sigma}^{\dagger} c_{j \alpha \sigma}-\frac{\Delta_{\text {cef }}}{2} \sum_{i \sigma}\left(n_{i 1 \sigma}-n_{i 2 \sigma}\right) \\
& +U \sum_{i \alpha} n_{i \alpha \uparrow} n_{i \alpha \downarrow}+U^{\prime} \sum_{i \sigma \sigma^{\prime}} n_{i 1 \sigma} n_{i 2 \sigma^{\prime}} \\
& -\frac{J}{2} \sum_{i}\left(n_{i 1 \uparrow}-n_{i 1 \downarrow}\right)\left(n_{i 2 \uparrow}-n_{i 2 \downarrow}\right),
\end{aligned}
$$

where $\langle i, j\rangle$ denotes the summation over the nearest neighbor sites, $c_{i \alpha \sigma}\left(c_{i \alpha \sigma}^{\dagger}\right)$ is an annihilation (creation) operator of an electron with spin $\sigma(=\uparrow, \downarrow)$ and orbital index $\alpha(=1,2)$ at the $i$ th site, and $n_{i \alpha \sigma}=c_{i \alpha \sigma}^{\dagger} c_{i \alpha \sigma} . t_{i j}$ is the transfer integral, $\Delta_{\text {cef }}$ is the crystal field splitting, $U\left(U^{\prime}\right)$ is the intraband (interband) Coulomb interaction, and $J$ is the Hund coupling. Here, we neglect translational symmetry breaking phases such as density waves, antiferromagnetically ordered or antiferroorbital ordered states. This para-magnetic/orbital solution should be essentially the same as that for the Hamiltonian with next-nearest-neighbor hopping $t^{\prime}$ equal to the nearest-neighbor hopping $t$ [24,25]. First, we do not impose any relations between the interactions, such as $U=U^{\prime}+5 / 2 J$. This allows us to discuss how the SC state, which is realized in the single band attractive Hubbard model $\left(U<0, U^{\prime}=J=0\right)$ is 
adiabatically connected to the strongly correlated SC state in model (1). Furthermore, we can discuss how the Hund coupling affects the SC state. We briefly address the SC state under the condition $U=U^{\prime}+5 / 2 J$ at the end.

To investigate the stability of the $s$-wave SC state, we use DMFT [9-12]. In DMFT, the original lattice model is mapped to an effective impurity model, which allows to take into account dynamical correlations. The lattice Green's function is obtained via a self-consistency condition imposed on the impurity problem. The DMFT treatment allows us to discuss the stability of the $s$-wave SC state more quantitatively than the static BCS mean-field theory [26]. In fact, the DMFT method has been successfully applied to various strongly correlated fermion systems with SC and superfluid states [27-34].

When the SC state is treated in the framework of DMFT, the Green's function should be described in the Nambu formalism. When the Cooper pairs are formed only in the same orbital, the impurity Green's function can be written as

$$
\hat{G}_{\mathrm{imp}, \alpha}(\tau)=\left(\begin{array}{cc}
G_{\alpha \uparrow}(\tau) & F_{\alpha}(\tau) \\
F_{\alpha}^{*}(\tau) & -G_{\alpha \downarrow}(-\tau)
\end{array}\right),
$$

where $G_{\alpha \sigma}(\tau)=-\left\langle T_{\tau} f_{\alpha \sigma}(\tau) f_{\alpha \sigma}^{\dagger}(0)\right\rangle$ denotes the normal Green's function for an electron with spin $\sigma$ and orbital $\alpha$, and $F_{\alpha}(\tau)=-\left\langle T_{\tau} f_{\alpha \uparrow}(\tau) f_{\alpha \downarrow}(0)\right\rangle$ and $F_{\alpha}^{*}(\tau)=$ $-\left\langle T_{\tau} f_{\alpha \downarrow}^{\dagger}(\tau) f_{\alpha \uparrow}^{\dagger}(0)\right\rangle$ denote the anomalous Green's functions. In the calculations, we use a semicircular density of states, $\rho(x)=2 /(\pi D) \sqrt{1-(x / D)^{2}}$, where $D$ is the half-bandwidth. The self-consistency equation is given by

$$
\hat{\mathcal{G}}_{\mathrm{imp}, \alpha}^{-1}\left(i \omega_{n}\right)=i \omega_{n} \hat{\sigma}_{0}+\mu \hat{\sigma}_{z}-\left(\frac{D}{2}\right)^{2} \hat{\sigma}_{z} \hat{G}_{\mathrm{imp}, \alpha}\left(i \omega_{n}\right) \hat{\sigma}_{z},
$$

where $\hat{\sigma}_{0}$ is the identity matrix and $\hat{\sigma}_{z}$ is the $z$ component of the Pauli matrix, $\omega_{n}=(2 n+1) \pi T$ is the Matsubara frequency, and $T$ is the temperature. $\hat{\mathcal{G}}_{\text {imp }}$ and $\hat{G}_{\text {imp }}$ are the noninteracting and full Green's functions for the effective impurity model. There are various techniques to solve the effective impurity problem. A particularly powerful method for exploring finite-temperature properties is the hybridizationexpansion CTQMC method [13,14]. It enables us to study the Hubbard model in both the weak- and strong-coupling regimes. The Green's functions are measured on a grid of more than one thousand points.

In this paper, we use the half bandwidth $D$ as the unit of energy. To examine the nature of the low-temperature states, we calculate the double occupancies $d_{\text {intra }}, d_{p}, d_{a}$, and the pair potential $\Delta_{\alpha}$, which are given by

$$
\begin{gathered}
d_{\text {intra }}^{(\alpha)}=\left\langle n_{i \alpha \uparrow} n_{i \alpha \downarrow}\right\rangle, \\
d_{p}=\frac{1}{2} \sum_{\sigma}\left\langle n_{i 1 \sigma} n_{i 2 \sigma}\right\rangle, \\
d_{a}=\frac{1}{2} \sum_{\sigma}\left\langle n_{i 1 \sigma} n_{i 2 \bar{\sigma}}\right\rangle, \\
\Delta_{\alpha}=\left\langle c_{i \alpha \uparrow} c_{i \alpha \downarrow}\right\rangle=\lim _{\tau \rightarrow 0_{+}} F_{\alpha}(\tau) .
\end{gathered}
$$

We also calculate the quantity $Z_{\alpha \sigma}=\left[1-\operatorname{Im} \Sigma_{\alpha \sigma}\left(i \omega_{0}\right) / \omega_{0}\right]^{-1}$ to estimate the quasiparticle weight at finite temperatures.
Here, $\Sigma_{\alpha \sigma}$ is the normal self-energy for spin $\sigma$ and orbital $\alpha$.

Before we start with discussions, let us mention some known facts about the Mott transitions in the paramagnetic system. When $U^{\prime}=0$, the system reduces to the single band Hubbard model. At zero temperature, the transition to the Mott insulating state occurs at $U / D \sim 3$ [35], while the pairing transition occurs at $U / D \sim-3$ [36]. When $U=U^{\prime}$, a Fermi liquid behavior is stabilized up to fairly large interactions due to orbital fluctuations, and the Mott transition occurs at $U / D \sim 5$ [21,37]. On the other hand, the system is driven to the Mott insulating state away from this condition [21,22]. In the following, we systematically study the appearance of a superconducting phase in this model, neglecting translational symmetry breaking phases. Then, we clarify the role of the Mott transitions in stabilizing the SC state.

\section{RESULTS}

In this section, we discuss the stability of the SC state in the half-filled two-band Hubbard model with $J=0$ and $\Delta_{\text {cef }}=0$. By combining DMFT with the CTQMC impurity solver, we obtain the pair potentials at $T / D=0.01$, as shown in Fig. 1 . When $U^{\prime}=0$, the two-band system is decoupled and becomes equivalent to the single-band Hubbard model. In this model, the SC ground state is realized only when the intraorbital interaction is attractive. At the finite temperature $T / D=0.01$, the SC state is realized in the intermediate coupling region $\left(U_{c 1}<U<U_{c 2}\right)$ due to thermal fluctuations, where $U_{c 1} / D \sim$ -26.8 and $U_{c 2} / D \sim-0.49$. In the weak-coupling region $U_{c 2}<U<0$, the system is metallic. Increasing the magnitude of the attractive interaction beyond the critical value $U_{c 2}$, weakly coupled Cooper pairs are formed and the BCS-type SC state is realized. In the strong-coupling region, two fermions are tightly coupled and composite bosons are condensed, so that a BEC-type SC state is realized at low temperatures. In this case, the energy scale should be $t^{2} /|U|$, characteristic of the hopping for paired bosons. Therefore a further increase in the attractive interaction effectively increases the temperature of the system and destabilizes the SC state. At $U=U_{c 1}$, the pair

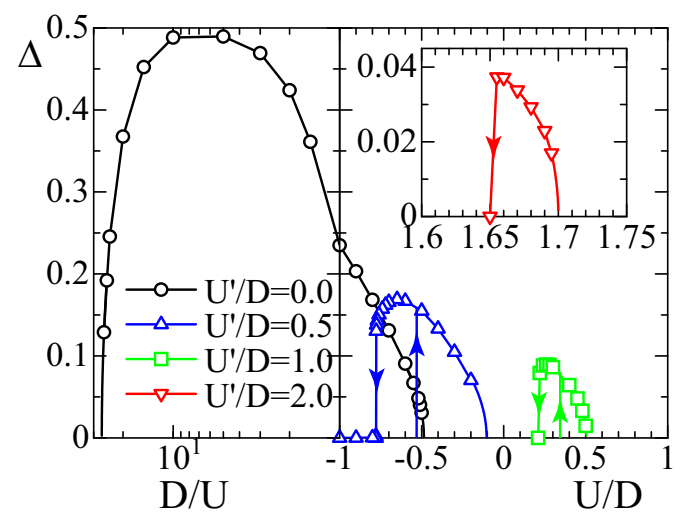

FIG. 1. (Color online) Pair potential $\Delta$ as a function of $U / D$ when $T / D=0.01$ and $J=\Delta_{\text {cef }}=0$. The arrows indicate the existence of a hysteresis in the pair potential. The inset shows the results for $U^{\prime} / D=2.0$. 
potential vanishes with a second-order phase transition to the normal state. It is known that the BCS-BEC crossover occurs between these regions. When $U<U_{c 1}$, the paramagnetic paired Mott (PM) state is realized, with an equal probability for doubly-occupied and empty states at half-filling [36].

Turning on the interband interaction $U^{\prime}$ does not simply destabilize the SC state, but leads to a remarkable behavior, as shown in Fig. 1. When the onsite interaction $U$ decreases with a fixed $U^{\prime} / D=0.5$, the conventional critical behavior appears around $U_{c 2} / D \sim-0.10$ in the BCS region. Since the introduction of the interorbital interaction $U^{\prime}$ increases the critical interaction $U_{c 2}$, we conclude that in the BCS region, the SC state becomes more stable. On the other hand, in the $\mathrm{BEC}$ region, we find a clear jump singularity at $U=U_{c 1}^{(s)}$ in the curve of the pair potential, where $U_{c 1}^{(s)} / D \sim-0.78$. This suggests the existence of a first-order phase transition to the PM state. In fact, as the intraorbital interaction $U$ is increased from the PM state, the phase transition occurs at $U=U_{c 1}^{(m)}$ together with a jump singularity in the curve of the pair potential, where $U_{c 1}^{(m)} / D \sim-0.53$. Therefore the nature of the phase transition in the BEC region is changed by the introduction of the interorbital interaction, in contrast to that in the BCS region. We also find that the first-order phase transition points are rapidly shifted to larger $U$, as shown in Fig. 1 . This can be explained by the fact that the interband Coulomb interactions stabilize the PM state, while they have little effect on the SC state. When $U^{\prime} / D=1.0$, we obtain the transition points $U_{c 1}^{(s)} \sim 0.22, U_{c 1}^{(m)} \sim 0.34$ and $U_{c 2} \sim 0.50$. We note that the $s$-wave SC state is realized even in the repulsively interacting region $\left(U, U^{\prime}>0\right)$ although the magnitude of the pair potential is rather small, as shown in Fig. 1. A further increase in the interorbital interaction decreases the pair potential until the SC state disappears in the large $U^{\prime}$ region (there is no SC solution for $U^{\prime} / D>3$ at $\left.T / D=0.01\right)$.

By performing similar calculations, we obtain the phase diagram at $T / D=0.01$ as shown in Fig. 2. In the negative $U$ region with a small $U^{\prime}$, the trivial SC state is stabilized by the

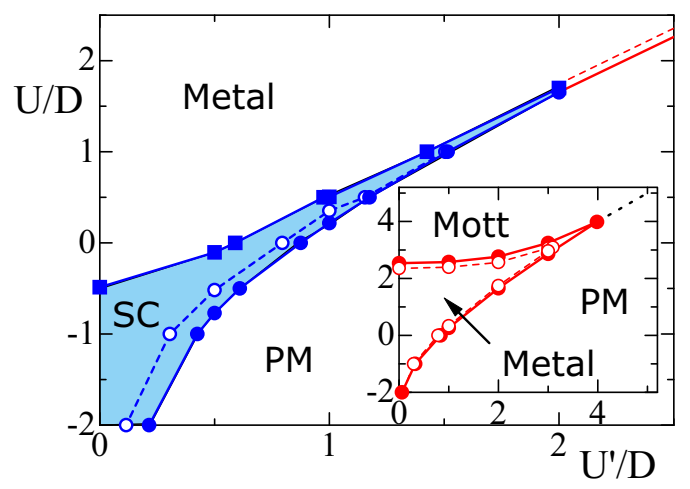

FIG. 2. (Color online) Phase diagram of the two-band Hubbard model when $T / D=0.01$ and $J=\Delta_{\text {cef }}=0$. Squares represent the second-order phase transition points $\left(U_{c 2}\right)$. Solid (open) circles represent the transition points $U_{c 1}^{(s)}\left(U_{c 1}^{(m)}\right)$, where the SC (PM) state disappears. The inset shows the paramagnetic phase diagram of the model. Solid (open) circles correspond to the transition points where the metallic (Mott) state disappears. The dotted line indicates the crossover line between the conventional Mott and PM states. intraorbital attractive interaction. The increase in the interband interaction $U^{\prime}$ shifts the phase boundaries to larger $U$ and the $\mathrm{SC}$ state is stabilized in a narrower parameter region. The critical end point is located around $\left(U / D, U^{\prime} / D\right) \sim(1.5,2.0)$. An important point is that the $s$-wave SC state appears even in the repulsively interacting region with $U^{\prime}>U>0$. This suggests that the interorbital interactions as well as the intraorbital interactions play a crucial role in stabilizing the $\mathrm{SC}$ state in this region. We note that within the SC region, no singularities appear in physical quantities such as double occupancies, renormalization factors, or pair potentials. This implies a crossover between the SC state stabilized by the on-site attraction $U(<0)$ and the SC state for $U^{\prime}>U>0$.

A similar behavior has been found in the three-component Hubbard model at half-filling [33]. In this case, the SC state appears along the first-order phase boundary between the metallic and PM states in the paramagnetic phase. However, the paramagnetic properties, and in particular the Mott physics, depend on the number of components for each fermion. For example, in the three component system, the Mott transition never occurs in the symmetric case $U=U^{\prime}(>0)$, while it occurs for the four component system equivalent to the degenerate Hubbard model (1). Therefore it is necessary to clarify how the competition between the metallic and Mott states in the paramagnetic phase affects the stability of the superconducting state.

For this purpose, we examine the paramagnetic properties at the temperature $T / D=0.01$, even though the ground-state phase diagram has already been calculated [21]. Figure 3 shows the renormalization factors and double occupancies. When we increase the intraorbital interaction $U$ with $U^{\prime}=0$, the conventional Mott transition occurs around $U / D=2.5$. In the strong coupling region with $U>U^{\prime}, d_{\text {intra }} \sim 0$ and $d_{p}=$ $d_{a} \sim 0.25$. This implies that each orbital is occupied by one fermion and the Mott insulating state is realized. In the strong coupling region $U / D(>2.8)$ with $U^{\prime} / D=2.0$, a similar behavior appears. On the other hand, when $U / D<1.6$ and $U^{\prime} / D=2.0, d_{\text {intra }} \sim 0.5$ and $d_{p}=d_{a} \sim 0.0$. This indicates that at each site we have a tightly coupled singlet (empty) state in one orbital, and an empty (tightly coupled singlet) state in the other. Therefore, in this region, the PM state is realized. In the symmetric case $U=U^{\prime}$, it is known that spin and orbital fluctuations are equally enhanced and the metallic state is stable up to a fairly large interaction [21]. The Mott transition occurs at $U / D \sim 4.0$, where $d_{\text {intra }}=d_{p}=d_{a} \sim 0.167$. Since the Mott phase transitions are of first order, a hysteresis appears in these quantities in Fig. 3. There is a crossover between two Mott states in the strong coupling region, as shown in the inset of Fig. 3.

By performing similar calculations in the $\left(U, U^{\prime}\right)$ plane, we obtain the paramagnetic phase diagram of the degenerate Hubbard model, as shown in the inset of Fig. 2, where the phase transition from the metallic (Mott) state occurs at the phase boundary shown with solid (dashed) lines. An important point is that the SC state discussed before is realized along the first-order phase boundary between the metallic and PM states (Fig. 2). This is similar to the finding for the three component system. Therefore we can conclude that charge fluctuations for the paired particles, which are induced by the density-density type interorbital interactions, play an essential 
(a)

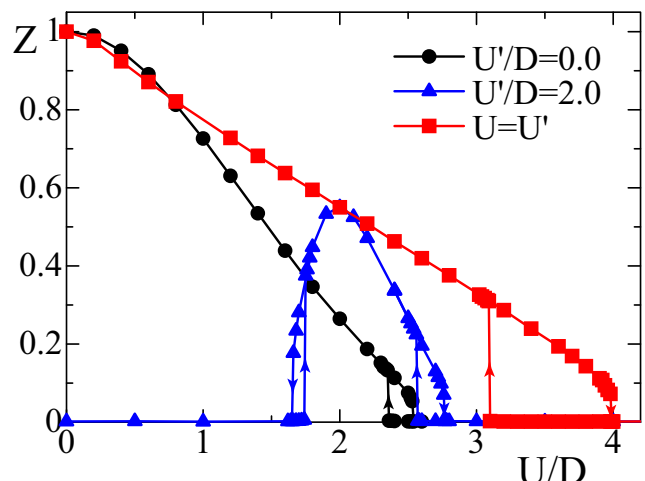

(b)

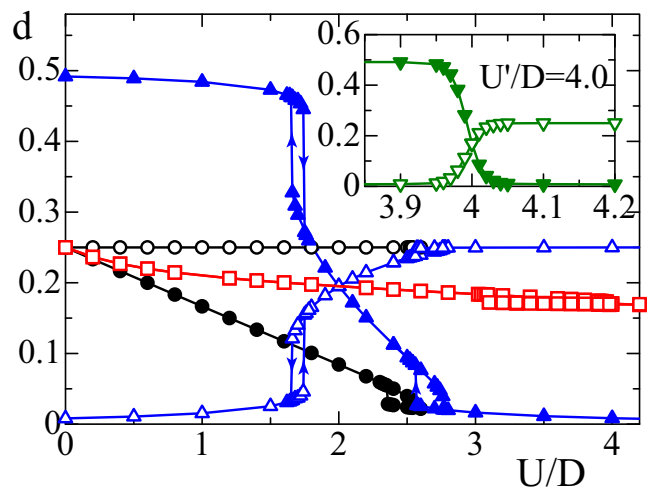

FIG. 3. (Color online) Renormalization factor (a) and double occupancy (b) as a function of the intraorbital interaction $U / D$ in the paramagnetic system at $T / D=0.01$ when the interactions are fixed as $J=\Delta_{\text {cef }}=0.0$ and $U^{\prime} / D=0.0,1.0,2.0$, and 3.0. Solid (open) symbols in (b) represent the double occupancy $d_{\text {intra }}\left[d_{\text {inter }}(=\right.$ $\left.d_{p}=d_{a}\right)$.

role in stabilizing the SC state. On the other hand, concerning the critical end point (CEP), there is a difference between these two systems. In the three component systems, the CEP for the SC state is located near the CEP for the Mott transitions at low temperatures [33]. On the other hand, in our model, the conventional Mott state is adiabatically connected to the PM state through the crossover. Therefore, at low temperatures, there is no CEP in the paramagnetic state. This results in the existence of the first order phase transition between the metallic and PM states beyond the CEP for the SC state, as shown in Fig. 2.

This competition leads to interesting finite-temperature properties. We show the finite-temperature phase diagrams for $U^{\prime} / D=0.5,1.0$, and 3.0 in Fig. 4. In our calculations, it is hard to determine quantitatively the first-order transition point where the free energies of the competing states cross. Therefore we show in the figure the coexisting region with competing solutions delimited by the open and full symbols. A crossover between the metallic and Mott states occurs at higher temperatures, which is roughly determined by the inflection point in the curve of the renormalization factor. When $U^{\prime} / D=0.5$, the SC state is realized over a wide $U$ range at low temperatures due to the strong attractive interaction $U$. The maximum of the critical temperature is located around $(U / D, T / D) \sim(-0.5,0.034)$. In this case, the Mott phase boundaries for the paramagnetic state are within the SC dome,
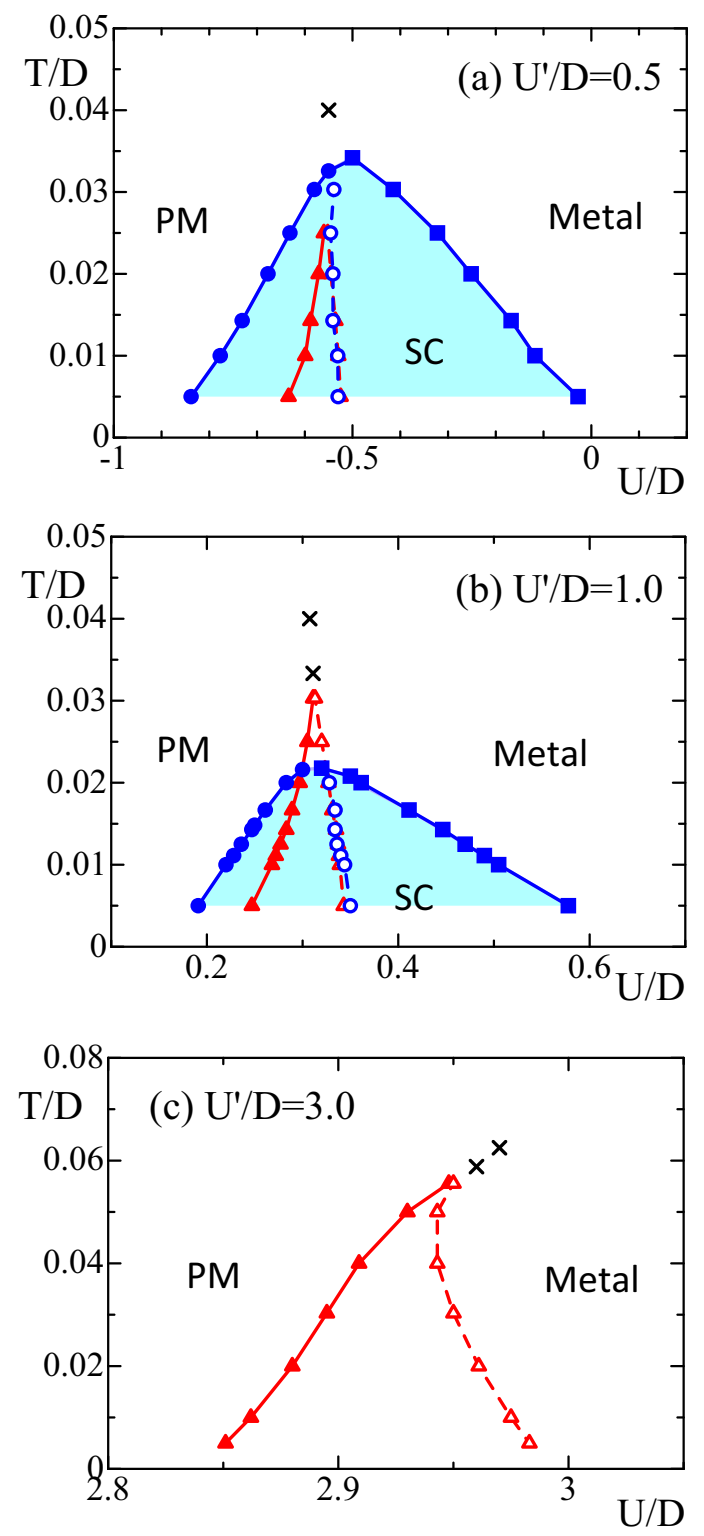

FIG. 4. (Color online) Finite-temperature phase diagrams of the two-band Hubbard model when $J=\Delta_{\text {cef }}=0$ and $U^{\prime} / D=0.5,1.0$, and 3.0. Squares represent the second-order phase transition points $\left(U_{c 2}\right)$. Solid (open) circles represent the transition point $U_{c 1}^{(s)}\left(U_{c 1}^{(m)}\right)$ and solid (open) triangles represent the transition point, where the metallic (PM) state disappears.

as shown in Fig. 4(a). Therefore no Mott transition occurs and the low-temperature properties are similar to those for the single band Hubbard model [25]. Increasing the interorbital interaction $U^{\prime}$, the SC region shrinks and the maximum of the critical temperature decreases. On the other hand, the critical temperature for the Mott phase transition shifts to higher temperature. This yields a phase diagram with both phase boundaries for the Mott and superconducting transitions, as shown in Fig. 4(b). The SC state is realized at low temperatures, while the Mott transition occurs for higher temperatures. We find that the Mott transition line appears around the maximum of the superconducting dome. This is consistent with the observation discussed above, that the SC state is realized 
along the first-order phase boundary between the metallic and PM states. A further increase of the interband interaction destabilizes the SC state, so that only the Mott transition line appears in the phase diagram, as shown in Fig. 4(c). Note that the critical temperature for the Mott transition increases when the system approaches the $\mathrm{SU}(4)$ point $\left(U=U^{\prime}\right)$, which is consistent with the results obtained by the self-energy functional theory [38].

Here, we have shown that the $s$-wave SC state can be realized in the repulsively interacting model with $U^{\prime}>U>0$. It has also been found that the SC state appears along the first-order phase boundary between the metallic and PM phases. This observation suggests that the SC state should be stable against small perturbations such as a Hund coupling and a crystalline electric field. In the following section, we focus on the SC state in the repulsively interacting region to discuss its stability.

\section{STABILITY OF THE SUPERCONDUCTING STATE}

In this section, we consider the effects of the Hund coupling and crystalline electric field, which may affect the stability of the SC state in realistic materials. A positive (negative) Hund coupling favors the parallel (antiparallel) spin state between orbitals, while the crystalline electric field favors the doubly occupied state in the lower orbital. Therefore these terms in the Hamiltonian may destabilize the SC state.

First, setting $\Delta_{\text {cef }}=0$ and fixing the intra- and interorbital interactions $U$ and $U^{\prime}$ to certain values, we discuss how the SC state is affected by the Hund coupling. Note that when the onsite interactions $\left(U, U^{\prime}\right.$, and $\left.J\right)$ are independently varied, the pair potential and chemical potential are invariant under the transformation $J \rightarrow-J$. Figure 5 shows the results for the pair potential at the temperature $T / D=0.01$. When $J=0$, the BEC-type (BCS-type) SC state is realized with a finite pair potential in the system with $U / D=0.3(0.4)$ and $U^{\prime} / D=1.0$, as discussed in the previous section. The introduction of the Hund coupling monotonically decreases the pair potential. Finally, the pair potential vanishes and a phase transition occurs to the normal state. More specifically, when $U / D=0.3(0.4)$, the phase transition is of first (second)

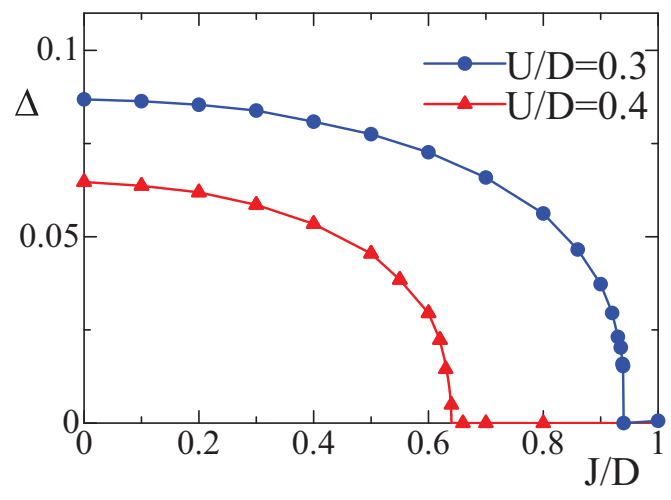

FIG. 5. (Color online) The pair potentials as a function of the Hund coupling $J$ in the two-band Hubbard model at $T / D=0.01$. Circles (triangles) represent the results for the system with $U / D=$ $0.3(0.4)$ and $U^{\prime} / D=1.0$.

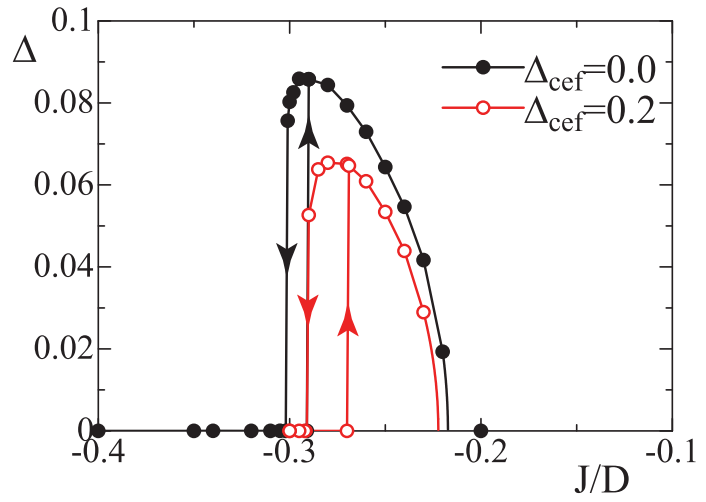

FIG. 6. (Color online) Pair potential as a function of the Hund coupling $J$ in the two-band Hubbard model with the condition $U=$ $U^{\prime}+5 / 2 J$ when $U / D=0.3$ and $T / D=0.01$.

order, which is consistent with the characteristic feature of the BEC (BCS) state. From the above results, we can say that the Hund coupling simply suppresses SC fluctuations.

On the other hand, if one considers the degenerate Hubbard model appropriate for realistic materials, the onsite interactions $\left(U, U^{\prime}, J\right)$ are not independent. Namely, when the Hund coupling $J$ is varied with a fixed intraorbital interaction $U$, the interorbital interaction $U^{\prime}$ is changed at the same time, which may induce the SC state. To clarify this point, we calculate the pair potential in the system under the condition $U=U^{\prime}+5 / 2 J$. When the Hund coupling $J$ is positive, the interorbital interaction $U^{\prime}$ is always smaller than the intraorbital one $U$ and the superconductivity is never realized. On the other hand, a negative Hund coupling may drive the system to the SC state since $U^{\prime}>U$. The results for the system with $U / D=0.3$ are shown in Fig. 6 .

First, we focus on the system without crystalline electric field. When the Hund coupling is small enough $(-0.2<J / D)$, the system is weakly correlated and the metallic state is realized. Increasing the magnitude of the Hund coupling, a second-order phase transition occurs to the SC state at $J_{c 2} / D=-0.22$. For $J$ below $J_{c}$, the pair potential grows and a SC state is stabilized. Finally, the first-order phase transition occurs at $J_{c 1}^{(s)} / D=-0.30$ with a jump singularity in the pair potential, as shown in Fig. 6. The double occupancy in each orbital approaches 0.5 and the other occupancies $d_{p}$ and $d_{a}$ approach zero when $J<J_{c 1}^{(s)}$. Therefore the PM state is realized in this region. The increase of the Hund coupling from the PM state induces another first-order phase transition at $J_{c 1}^{(m)} / D=-0.29$. The obtained results are essentially the same as those discussed in the previous section. Therefore we can say that the Hund coupling has little effect on the realization of the superconductivity. The introduction of the crystalline electric field increases (decreases) the electron number for the orbital 1 (2), which suppresses SC fluctuations. In fact, we find the decrease of the pair potential in the intermediate region $(J / D \sim-0.25)$ in Fig. 6 .

By performing similar calculations under the constraint $U=U^{\prime}+5 / 2 J$, we obtain the phase diagram in the space of $J$ and $\Delta_{\text {cef }}$, as shown in Fig. 7. One finds that the metallic, $\mathrm{SC}$, and PM states are stable against a small crystalline electric field. In the large $\Delta_{\text {cef }}$ case, the lower orbital should be 


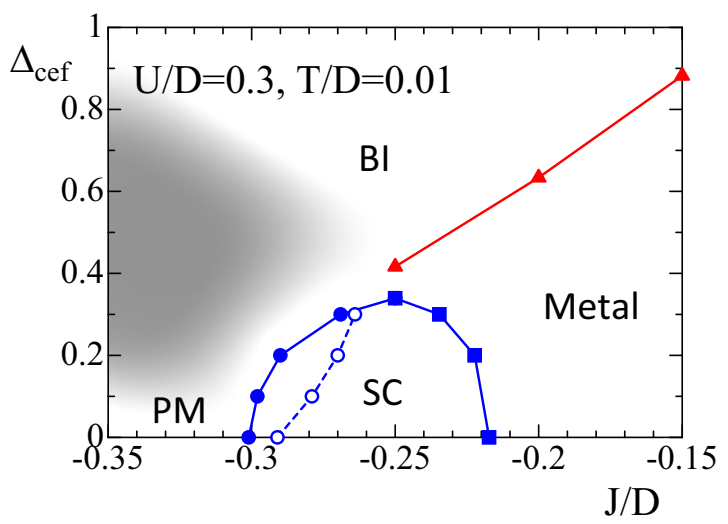

FIG. 7. (Color online) Phase diagram in the space of $J / D$ and $\Delta_{\text {cef }} / D$. Open (solid) circles represent the transition point $J_{c 1}^{(m)}\left(J_{c 1}^{(s)}\right)$ and solid squares represent the second-order transition point $J_{c 2}$. Solid triangles represent the boundaries between the band insulating (BI) and metallic states. In the shaded area, we could not obtain reliable results due to an inefficient Monte Carlo sampling.

fully occupied leading to a band insulator. The competition between the PM and band insulating states in the paramagnetic region is difficult to pin down since the Monte Carlo sampling with standard updates becomes inefficient. Therefore, the boundary between these two states could not be determined quantitatively. The $s$-wave SC state appears between the metallic, PM, and band insulating states. Figure 8 shows the density of states for the system with $U / D=0.3$ and $\Delta_{\text {cef }} / D=$ 0.2 at $T / D=0.01$. When $J / D=-0.2$, the weakly correlated metallic state is realized and no remarkable peak structures appear in the density of states. When $J / D=-0.25$, a tiny gap appears around the Fermi level, which indicates that the BCStype SC state is realized. In the PM state $(J / D=-0.3)$, tightly coupled singlet states are formed with $n_{1}>0.5$ and $n_{2}<0.5$. Therefore a large gap appears in the one-particle spectral function. This contrast with the conventional Mott insulating state, which is stabilized only at a commensurate filling.

Before closing this section, we briefly comment on the limitations of our DMFT treatment. Since this method takes into account local electron correlations, we can only discuss the stability of the $s$-wave SC state. Our calculations have clarified that the $s$-wave SC state is realized in the degenerate Hubbard model when $U^{\prime}>U$, at least if magnetic and/or orbital ordering is suppressed. In transition-metal oxides, the intra- and interorbital interactions usually satisfy the relation $U>U^{\prime}$, meaning that it is hard to realize the SC state discussed here. In this case, by taking into account the details of the band structure and intersite correlations, one has to discuss low-temperature phenomena such as unconventional SC with anisotropic gap functions, magnetically ordered or orbitally ordered states. On the other hand, if one considers

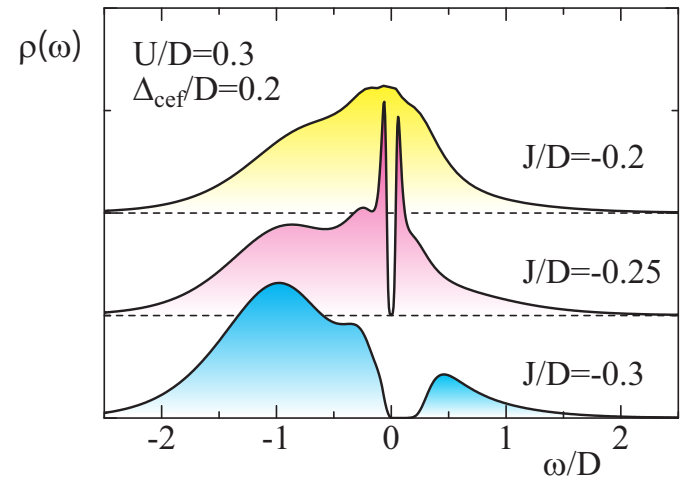

FIG. 8. (Color online) Density of states for electrons in orbital 1 in the system with $U / D=0.2$ and $\Delta_{\text {cef }} / D=0.2$ when $J / D=-0.2$, -0.25 , and -0.3 .

low-energy states of systems with a coupling to other degrees of freedom, the condition $U^{\prime}>U$ can be effectively realized. In the fullerene-based solid $\mathrm{A}_{3} \mathrm{C}_{60}$, it has been reported that this relation should be realized due to the strong electron-phonon coupling $[7,8,39,40]$. In such a situation, DMFT should capture the essence of the SC state observed in the material.

\section{SUMMARY}

We have investigated the low-temperature properties of the two-band Hubbard model with degenerate orbitals. By combining dynamical mean-field theory with continuous-time quantum Monte Carlo simulations, we have clarified that a SC state can be realized in a repulsively interacting twoorbital system due to the competition between the intra- and interorbital Coulomb interactions. In particular, this $s$-wave superconducting state appears along the first-order phase boundary between the metallic and paired Mott states in the para-magnetic/orbital system. On the other hand, the Ising-type Hund coupling destabilizes the SC state. Although we have not investigated the effect of the exchange and pair hopping parts of the Hund coupling term, we believe that these have little effect on the stability of the superconductivity. It will be interesting to clarify this point in a future investigation.

\section{ACKNOWLEDGMENTS}

The authors thank S. Suga and S. Hoshino for valuable discussions. This work was partly supported by the Grantin-Aid for Scientific Research from JSPS, KAKENHI No. 25800193 (A.K.). Some of computations in this work has been done using the facilities of the Supercomputer Center, the Institute for Solid State Physics, the University of Tokyo. The simulations have been performed using some of the ALPS libraries [41].
[1] Y. Maeno, H. Hashimoto, K. Toshida, S. Nishizaki, T. Fujita, J. G. Bednorz, and F. Lichtenberg, Nature (London) 372, 532 (1994).

[2] Y. Kamihara, T. Watanabe, M. Hirano, and H. Hosono, J. Am. Chem. Soc. 130, 3296 (2008).
[3] K. Takada, H. Sakurai, E. Takayama-Muromachi, F. Izumi, R. A. Dilanian, and T. Sasaki, Nature (London) 422, 53 (2003).

[4] A. F. Hebard, M. J. Rosseinsky, R. C. Haddon, D. W. Murphy, S. H. Glarum, T. T. M. Palstra, A. P. Ramirez, and A. R. Kortan, Nature (London) 350, 600 (1991). 
[5] K. Tanigaki, T. W. Ebbesen, S. Saito, J. Mizuki, J. S. Tsai, Y. Kubo, and S. Kuroshima, Nature (London) 352, 222 (1991).

[6] A. Y. Ganin, Y. Takabayashi, Y. Z. Khimyak, S. Margadonna, A. Tamai, M. J. Rosseinsky, and K. Prassides, Nat. Mat. 7, 367 (2008).

[7] M. Capone, M. Fabrizio, C. Castellani, and E. Tosatti, Science 296, 2364 (2002).

[8] M. Capone, M. Fabrizio, C. Castellani, and E. Tosatti, Phys. Rev. Lett. 93, 047001 (2004).

[9] W. Metzner and D. Vollhardt, Phys. Rev. Lett. 62, 324 (1989).

[10] E. Müller-Hartmann, Z. Phys. B 74, 507 (1989).

[11] A. Georges, G. Kotliar, W. Krauth, and M. J. Rozenberg, Rev. Mod. Phys. 68, 13 (1996).

[12] T. Pruschke, M. Jarrell, and J. K. Freericks, Adv. Phys. 44, 187 (1995).

[13] E. Gull, A. J. Millis, A. N. Rubtsov, A. I. Lichtenstein, M. Troyer, and P. Werner, Rev. Mod. Phys. 83, 349 (2011).

[14] P. Werner, A. Comanac, L. deMedici, M. Troyer, and A. J. Millis, Phys. Rev. Lett. 97, 076405 (2006).

[15] T. Momoi and K. Kubo, Phys. Rev. B 58, R567 (1998).

[16] M. J. Rozenberg, Phys. Rev. B 55, R4855 (1997).

[17] K. Held and D. Vollhardt, Eur. Phys. J. B 5, 473 (1998).

[18] J. E. Han, M. Jarrell, and D. L. Cox, Phys. Rev. B 58, R4199 (1998).

[19] V. S. Oudovenko and G. Kotliar, Phys. Rev. B 65, 075102 (2002).

[20] G. Kotliar and H. Kajueter, Phys. Rev. B 54, R14221 (1996).

[21] A. Koga, Y. Imai, and N. Kawakami, Phys. Rev. B 66, 165107 (2002).

[22] A. Koga, T. Ohashi, Y. Imai, S. Suga, and N. Kawakami, J. Phys. Soc. Jpn. 72, 1306 (2003).

[23] P. Werner and A. J. Millis, Phys. Rev. Lett. 99, 126405 (2007).
[24] R. Chitra and G. Kotliar, Phys. Rev. Lett. 83, 2386 (1999).

[25] R. Zitzler, T. Pruschke, and R. Bulla, Eur. Phys. J. B 27, 473 (2002).

[26] A. Georges, G. Kotliar, and W. Krauth, Z. Phys. B 92, 313 (1993).

[27] A. Garg, H. R. Krishnamurthy, and M. Randeria, Phys. Rev. B 72, 024517 (2005).

[28] T.-L. Dao, M. Ferrero, A. Georges, M. Capone, and O. Parcollet, Phys. Rev. Lett. 101, 236405 (2008).

[29] N. Takemori and A. Koga, J. Phys. Soc. Jpn. 81, 063002 (2012).

[30] O. Bodensiek, R. Zitko, M. Vojta, M. Jarrell, and T. Pruschke, Phys. Rev. Lett. 110, 146406 (2013).

[31] S. Hoshino and Y. Kuramoto, Phys. Rev. Lett. 112, 167204 (2014).

[32] A. Koga and P. Werner, J. Phys. Soc. Jpn. 79, 064401 (2010); 79, 114401 (2010); Phys. Rev. A 84, 023638 (2011).

[33] Y. Okanami, N. Takemori, and A. Koga, Phys. Rev. A 89, 053622 (2014).

[34] K. Inaba and S. I. Suga, Phys. Rev. Lett. 108, 255301 (2012).

[35] R. Bulla, Phys. Rev. Lett. 83, 136 (1999).

[36] M. Capone, C. Castellani, and M. Grilli, Phys. Rev. Lett. 88, 126403 (2002).

[37] Y. Ono, M. Potthoff, and R. Bulla, Phys. Rev. B 67, 035119 (2003).

[38] K. Inaba, A. Koga, S. I. Suga, and N. Kawakami, Phys. Rev. B 72, 085112 (2005).

[39] J. E. Han, O. Gunnarsson, and V. H. Crespi, Phys. Rev. Lett. 90, 167006 (2003).

[40] Y. Nomura and R. Arita (private communication).

[41] A. F. Albuquerque et al., J. Magn. Magn. Mater. 310, 1187 (2007). 